\title{
Study on Forsythin promoting apoptosis of laryngeal carcinoma cells by regulating miRNA-1469
}

\author{
Zhaomeng Guo ${ }^{1}$, Peng Zhao ${ }^{1}$, Xiaojia Zhu², Futang Wen³ ${ }^{3}$ Jiangqi Liu ${ }^{1}$, Shuqi Qiu ${ }^{1 \star}$ (D)
}

\begin{abstract}
Objective: The paper aimed to explore the mechanism of Forsythin regulating miRNA expression in laryngeal carcinoma cells, and to clarify the molecular biological mechanism of Forsythin regulating miRNA to promote the apoptosis of laryngeal carcinoma cells, providing theoretical and experimental basis for clinical application of Forsythin as an anti-laryngeal cancer treatment drug. Methods: A miR-1469 low-expression laryngeal carcinoma cell line was established. Western blot and flow cytometry were applied to detect the effect of Forsythin on cell apoptosis. Western blot was employed to detect the effects of Forsythin on P53 protein, P53 low expression, and P53 overexpression in laryngeal carcinoma cells, as well as the effects on overexpression of miRNA-1469, and on double low expression of P53 and Mcl1. Real-time PCR method was used to detect the effect of miR1469 on p53 low expression in laryngeal carcinoma cells. Results: Flow cytometry detection of cell apoptosis showed that, after the cells with low miR-1469 expression were treated with Forsythin, the apoptosis rate was significantly reduced. Western blot detection showed that, compared with the Control group, the expression level of miR-1469 was significantly reduced after Forsythin administration in Hep2 cells with low expression of P53. Compared with the idle Control group, the apoptosis level of laryngeal carcinoma cells in Hep2 cells with low expression of P53 was significantly reduced. In Hep2 cells transfected with P53 overexpression plasmids, apoptosis level of laryngeal carcinoma cells increased. Compared with the idle Control group, the apoptosis level of laryngeal carcinoma cells in the single-transformed P53 shRNA group decreased, while the apoptosis level of the double-transformed miR-1469 mimic+P53 shRNA group increased again. After drug treatment, the apoptosis level of the single-transformed P53 shRNA group decreased, while the apoptosis level of the double-transformed Mcl1 shRNA+P53 shRNA group increased again. Conclusion: Forsythin can promote the apoptosis of laryngeal carcinoma cells by up-regulating the expression of miR-1469 and then down-regulating the expression of Mcl1. The drug can up-regulate the expression of miR-1469 by elevating the expression of P53. miR-1469 can promote the apoptosis of laryngeal carcinoma cells by inhibiting the expression of its downstream target gene Mcl1.
\end{abstract}

Keywords: forsythin; miRNA-1469; laryngeal carcinoma cells; apoptosis.

Practical Application: Forsythin promoting apoptosis of laryngeal carcinoma cells.

\section{Introduction}

Laryngeal cancer is a common malignant tumor of the head and neck. It is the second most common malignant tumor of the respiratory tract. The main causes of laryngeal cancer are smoking, drinking, air pollution, occupational factors, and papilloma virus infection. The incidence rate of man to woman is approximately $5: 1$. With the increasing aging of the population, the high smoking rate and air pollution and other factors, the incidence of laryngeal cancer in China is not optimistic (Obid et al., 2019). Currently, although the surgery, radiotherapy and chemotherapy and targeted therapy technology for laryngeal cancer have made significant progress, the five-year survival rate is still around $60.9 \%$, with no significant improvement over the past (Jaiswal et al., 2019). Therefore, it is still of great importance to find new therapeutic targets and methods for the treatment of laryngeal cancer.

MircoRNAs are small non-coding RNAs with a length of about 18-25 nt. They can degrade mRNA or inhibit mRNA translation by binding to the 3'non-coding region of downstream target gene mRNA, thereby inhibiting the expression of target genes at the post-transcriptional level. Current research has proved that miRNA is involved in important biological processes such as cell development, differentiation, proliferation, and apoptosis (Zhang et al., 2018). Among them, miRNAs play an important role in the occurrence and development of tumors. Different miRNAs, some as proto-oncogenes and some as tumor suppressor genes, can regulate the proliferation, invasion and apoptosis of tumor cells (Liu et al., 2017; Sun et al., 2017). 


\section{Methods}

1. Flow cytometry to detect the effect of Forsythin on hep-2 cell apoptosis.

1.1 The effect of Forsythin on hep-2 cell apoptosis.

Hep- 2 cells were treated with $80 \mu \mathrm{M}$ Forsythin for $0 \mathrm{~h}, 24 \mathrm{~h}$ and $48 \mathrm{~h}$, respectively. After digestion, the prepared $1 \times$ Annexin $\mathrm{V}$ binding solution was added. $100 \mu \mathrm{l}$ of the above suspension was obtained and added with $5 \mu \mathrm{l}$ of Annexin V-FITC, $5 \mu \mathrm{l}$ of propidium iodide staining solution, and $400 \mu \mathrm{l}$ of $1 \times$ Annexin $\mathrm{V}$ binding solution successively. Following this, flow cytometry was used for cell apoptosis detection within $1 \mathrm{~h}$.

1.2 Flow cytometry to detect the pro-apoptotic effect of Forsythin on miRNA-1469 low-expression in laryngeal carcinoma cells.

Hep-2 cells in the logarithmic growth phase were inoculated into a 6-well plate and cultured for 24 hours. Transfection operation was performed according to the instructions of LipofectamineTM2000 reagent. The cells were transfected with miR-1469 inhibitor for 24-36 hours, and an empty Control group was set. The original cell culture medium was removed. The cells were added with $20 \mu \mathrm{l}$ of $80 \mu \mathrm{M}$ Forsythin for $48 \mathrm{~h}$ treatment, while the Control group cells were added with $20 \mu \mathrm{l}$ ethanol solvent for $48 \mathrm{~h}$ administration. The detection method is as above.

2. Western blot detection of the effects of Forsythin on various proteins in laryngeal carcinoma cells.

2.1 Western blot detection of the pro-apoptotic effect of Forsythin on laryngeal carcinoma cells with low expression of miRNA-1469.

The expression of PARP protein was detected according to Western blot method.

2.2 Western blot determination of the effect of Forsythin on the expression of P53 protein in laryngeal carcinoma cells.

The expression of P53 protein was detected according to Western blot method.

2.3 Western blot to detect the effect of Forsythin on the expression of P53 in laryngeal carcinoma cells stably transfected with pLKO.1-P53shRNA, and to detect the transfection efficiency.

The expression of P53 protein was detected according to Western blot method.

2.4 Western blot detection of the effect of Forsythin on the apoptosis of laryngeal carcinoma cells with low expression of P53.

The expression of P53 and PARP proteins were detected according to Western blot method.

2.5 Western blot detection of the effect of Forsythin on the apoptosis of P53 overexpression in laryngeal carcinoma cells.

The expression of P53 and PARP proteins were detected according to Western blot method.
2.6 Western blot to detect the effect of Forsythin on the apoptosis of cells with P53 low expression and miRNA-1469 overexpression.

The expression of PARP protein was detected according to Western blot method.

2.7 Western blot to detect the effect of Forsythin on the apoptosis of cells with low expression of P53 and Mcl1.

The expression of PARP protein was detected according to Western blot method.

\section{Statistical analysis.}

All experiments were repeated three times independently. Western blot and flow cytometry images were the representative results. The experimental data are expressed as the mean \pm standard deviation, and the data is analyzed by one-way ANOVA. $\mathrm{P}<0.05$ indicates that the results are statistically different.

\section{Result}

\subsection{Forsythin can promote the apoptosis of laryngeal carcinoma cells}

Flow cytometry was used to detect cell apoptosis, and the results showed that the apoptosis rate of Hep-2 cells gradually increased with the prolongation of Forsythin administration, as shown in Figure 1.

The apoptosis effect of Forsythin on Hep-2 cells transfected with miR-1469 inhibitor and blank plasmid was detected by flow cytometry. The results showed that, compared with the blank Control group, the apoptosis rate of cells transfected with miR-1469 inhibitor significantly declined, proving that down-regulating the expression of miR-1469 can inhibit the pro-apoptotic effect of Forsythin on laryngeal carcinoma cells, as shown in Figure 2.

\subsection{The expression of Forsythin on various proteins}

Western blot was employed to detect the expression of p53 protein in Hep 2 cells after Forsythin treatment for different hours. It is found that, with the prolongation of Forsythin administration, p53 expression increased, as shown in Figure 3.

In order to clarify whether the up-regulation of miR-1469 expression by Forsythin is related to the up-regulation of p53 and PARP expression, Hep2 cells stably transfected with p53 shRNA were treated with Forsythin for 48 hours. The expression of $\mathrm{p} 53$ was measured to calculate the transfection efficiency. The results showed that, p53 protein in Hep2 cells stably transfected with P53 shRNA did not increase with the treatment of Forsythin. The expression level of PARP splicing protein in Hep2 cells transfected with p 53 shRNA was significantly reduced, indicating that the pro-apoptotic effect of Forsythin on laryngeal carcinoma cells is inhibited. See Figures 4 and 5.

By the same principle, Hep2 cells transfected with p53 overexpression plasmid and blank plasmid were treated with the drug for $48 \mathrm{~h}$. The results showed that, compared with the Control group, the expression level of PARP splicing protein 


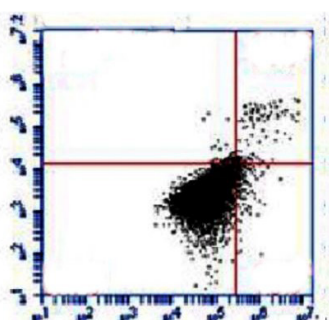

$0 \mathrm{~h}$

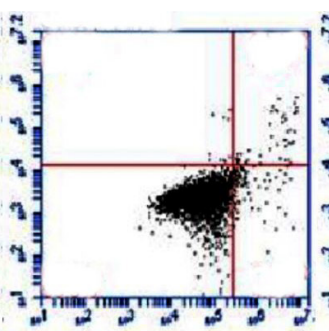

$24 \mathrm{~h}$

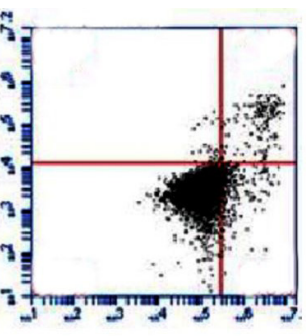

$48 \mathrm{~h}$

Figure 1. Flow cytometry to detect the effect of Forsythin on hep-2 cell apoptosis. $80 \mu \mathrm{M} 80 \mu \mathrm{M}+\mathrm{miR}-1469$ inhibitor.
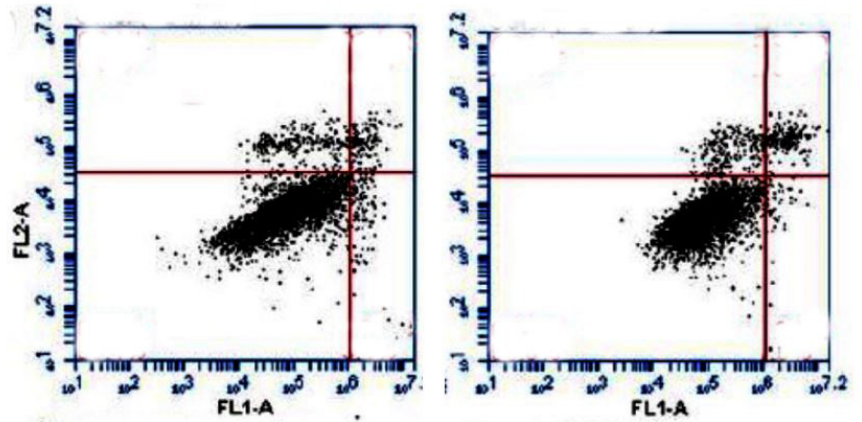

$0 \mathrm{~h}$
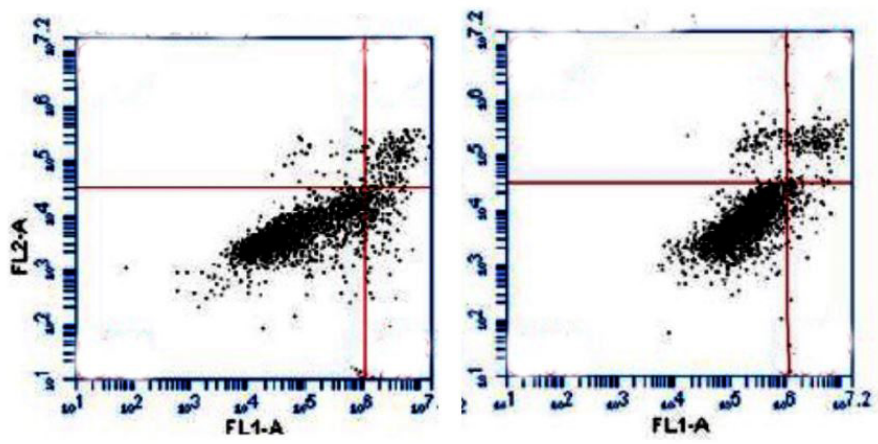

$48 \mathrm{~h}$

Figure 2. Down-regulating the expression of miR-1469 can inhibit the pro-apoptotic effect of Forsythin on laryngeal carcinoma cells.

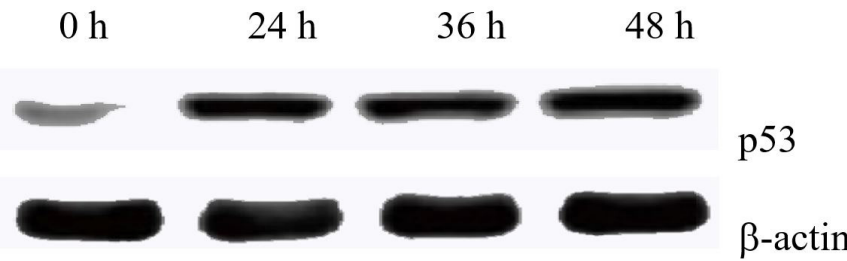

Figure 3. The effect of Forsythin on the expression of $\mathrm{p} 53$ protein.

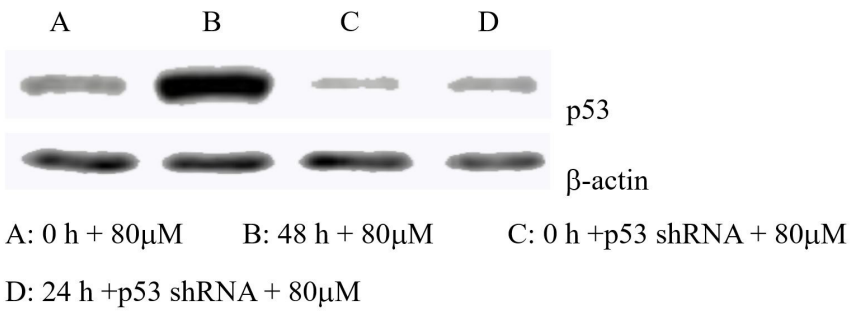

Figure 4. The effect of Forsythin on p53 protein in Hep2 cells stably transfected with p53 shRNA. in Hep 2 cells transfected with p53 overexpression plasmid increased, indicating that the pro-apoptotic effect of Forsythin on laryngeal carcinoma cells is enhanced, see Figure 6.

In order to determine whether Forsythin up-regulates the expression of miR-1469 by up-regulating the expression of P53, thus playing a role in promoting the apoptosis of laryngeal carcinoma cells, we designed a rescue experiment. We set up a control group, a single-transformed p53 shRNA group, and a double-transformed miR-1469 mimic + p53 shRNA group. After Forsythin administration for 48 hours, the results showed that, compared with the blank control group, the expression of PARP splicing protein in the single-transformed p53 shRNA group was reduced, indicating that apoptosis was inhibited. However, the expression of PARP splicing protein in the double-transformed miR-1469 mimic + p53 shRNA group increased again, indicating up-regulating the expression of miR-1469 can reverse the inhibitory effect of 553 shRNA on apoptosis, further proving that Forsythin can promote the apoptosis of laryngeal carcinoma cells by up-regulating the expression of P53 and then up-regulating the expression of miR-1469, as shown in Figure 7. 


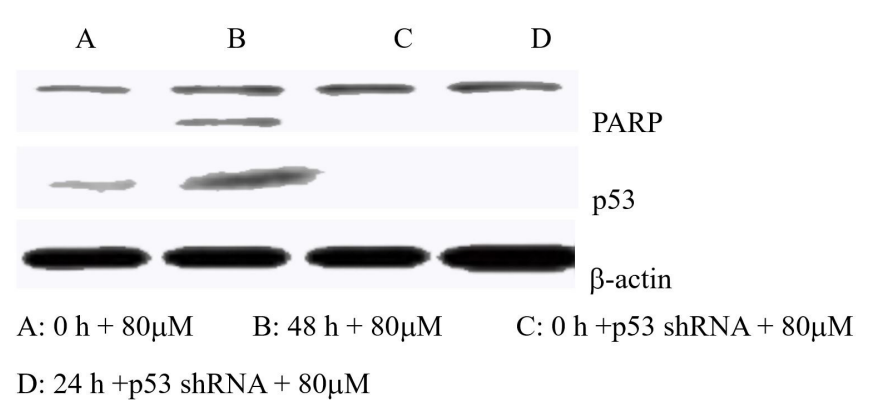

Figure 5. Down-regulating the expression of p53 can inhibit the pro-apoptotic effect of Forsythin on laryngeal carcinoma cells.

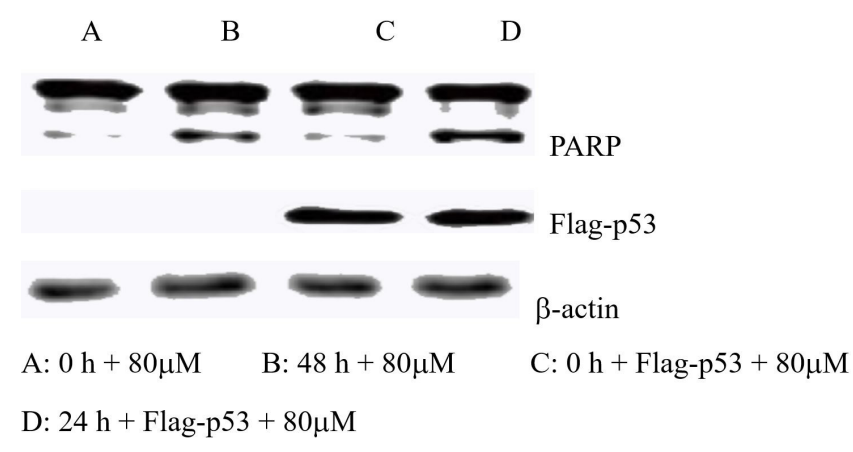

Figure 6. Up-regulation of $\mathrm{p} 53$ expression can promote the pro-apoptotic effect of Forsythin on laryngeal carcinoma cells.

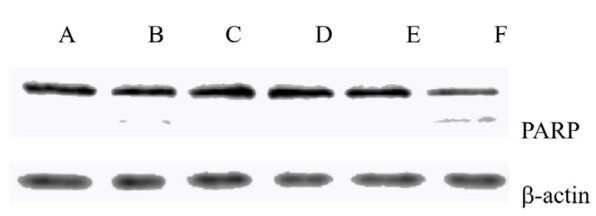
A: $0 \mathrm{~h}+80 \mu \mathrm{M} \quad$ B: $48 \mathrm{~h}+80 \mu \mathrm{M}$
$\mathrm{C}: 0 \mathrm{~h}+\mathrm{p} 53$ shRNA $+80 \mu \mathrm{M}$
D: $24 \mathrm{~h}+\mathrm{p} 53$ shRNA $+80 \mu \mathrm{M} \quad \mathrm{E}: 0 \mathrm{~h}+\mathrm{p} 53$ shRNA $+80 \mu \mathrm{M}+\mathrm{miR}-1469$
F: $0 \mathrm{~h}+\mathrm{p} 53$ shRNA $+80 \mu \mathrm{M}+\operatorname{miR}-1469$

Figure 7. Up-regulating the expression of miR-1469 can reverse the inhibitory effect of Forsythin promoting the apoptosis of laryngeal carcinoma cells caused by low p53 expression.

In order to clarify whether Forsythin regulates the miR-1469/ Mcl1 pathway by up-regulating the expression of $\mathrm{p} 53$, thus playing the role of promoting the apoptosis of laryngeal carcinoma cells, we designed another a rescue experiment. We set up a control group, a single-transformed p53 shRNA group, and a double-transformed Mcl1 shRNA + p53 shRNA group. After 48 hours of Forsythin administration, it was found that, compared with the blank Control group, the expression of PARP splicing protein in the single-transformed p53 shRNA group decreased, indicating that apoptosis was inhibited. However, the expression of PARP splicing protein in the double-transformed Mcl1 shRNA+p53 shRNA group increased again, suggesting down-regulating the expression of Mcll can reverse the inhibitory effect of p53 shRNA on apoptosis, further proving that Forsythin up-regulates the expression of p53 and then up-regulates the expression of miR-1469. miR-1469 inhibits the expression of its

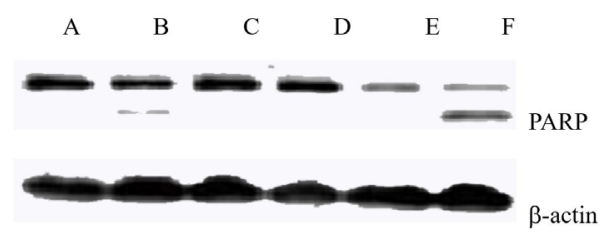
A: $0 \mathrm{~h}+80 \mu \mathrm{M}$
$\mathrm{B}: 48 \mathrm{~h}+80 \mu \mathrm{M}$
$\mathrm{C}: 0 \mathrm{~h}+\mathrm{p} 53$ shRNA $+80 \mu \mathrm{M}$
$\mathrm{D}: 24 \mathrm{~h}+\mathrm{p} 53$ shRNA $+80 \mu \mathrm{M}$
$\mathrm{E}: 0 \mathrm{~h}+\mathrm{p} 53$ shRNA $+80 \mu \mathrm{M}+\mathrm{Mcl} 1$
$\mathrm{F}: 0 \mathrm{~h}$
+ p5 3 shRNA $+80 \mu \mathrm{M}+\mathrm{Mcl} 1$

Figure 8. Down-regulation of Mcl1 expression can reverse the inhibitory effect Forsythin promoting the apoptosis of laryngeal carcinoma cells induced by $\mathrm{P} 53$ low expression.

downstream target gene Mcl1, thereby promoting the apoptosis of laryngeal carcinoma cells. See Figure 8.

\section{Discussion}

The $\mathrm{p} 53$ protein has a regulatory effect on the expression of miRNA. This regulation is not only at the level of miRNA transcription, but also at the level of processing after miRNA transcription. At the transcriptional level, studies have shown that $\mathrm{p} 53$ has binding sites in the promoter regions of miR-34a and miR-200c. By binding to this region, p53 activates the transcription process of miR-34a and miR-200c, thereby up-regulating miR-34a and miR-200c expressions (Yang et al., 2017a, b). Studies have found that p53 can up-regulate the expression of miR-29c in colon carcinoma cells, and miR-29c can act on its downstream target gene PHLDB2 to down-regulate its expression, thereby inhibiting the invasion and metastasis of colon carcinoma cells. By contrast, the binding site of $\mathrm{p} 53$ and miR-17-92 promoter region covers the TATA box region, thus interfering with the binding of TATA box binding protein (TBP) with TATA box, thereby inhibiting the transcription of miR-17-92 and down-regulating its expression level (Zhang et al., 2018a; Zhang et al., 2018b; Zhang et al.,2018c).

At the post-transcriptional processing level of miRNA, under the action of RNA polymerase II, the miRNA gene is transcribed to form the initial product pri-miRNA, which is about 300 1000 bases in length (Sun et al., 2017a, b). The pri-miRNA undergoes processing of ribonuclease III Drosha, becomes the pre-miRNA, or the microRNA precursor, with a length of about 70 to 90 bases (Zhao et al., 2017). The pre-miRNA is cleaved by another ribonuclease III Dicer to form mature miRNA at a length of about 18-23 nt (Tron et al., 2018; Chen et al., 2017). Studies have found that $\mathrm{p} 53$ protein can promote the processing and maturation of some miRNAs after transcription. It is worth noting that this part of miRNAs are mainly the ones that play a tumor suppressor effect. The specific mechanism is that p53 protein acts on Drosha enzymes to promote pri-miRNAs to transform into miRNA precursors. The transformation (of pre-miRNA) ultimately promotes the increase of mature miRNA expression. The p53 gene up-regulates the expression of miR-16-1, miR-143 and miR-145 through this mechanism.

In order to clarify whether Forsythin up-regulating the expression of miR-1469 in laryngeal carcinoma cells is related to the p53 protein, we used the Hep2 cell line with low p53 expression as 
the target. Forsythin up-regulating the expression of miR-1469 is related to it up-regulating the expression of $\mathrm{p} 53$ protein, indicating that Forsythin can up-regulate the expression of miR-1469 through elevating the expression of p53 in laryngeal carcinoma cells. So, does the change of p53 expression affect the effect of Forsythin in promoting the apoptosis of laryngeal carcinoma cells? In this regard, we used western blot to detect the effect of Forsythin on the apoptosis of $\mathrm{p} 53$ overexpression and p53 low expression in laryngeal carcinoma cells. The results showed that, the expression level of PARP splicing protein in laryngeal carcinoma cells with low p53 expression was significantly reduced, proving that the pro-apoptotic effect of Forsythin on laryngeal carcinoma cells was inhibited. However, the expression level of PARP splicing protein in laryngeal carcinoma cells with high p53 expression was increased. This indicates that the pro-apoptotic effect of Forsythin on laryngeal carcinoma cells is enhanced, proving that Forsythin can promote the apoptosis of laryngeal carcinoma cells by up-regulating the expression of P53.

On this basis, in order to clarify whether Forsythin can promote the apoptosis of laryngeal carcinoma cells through the P53/miR1469/Mcl1 signaling pathway, we designed a rescue experiment. First, on the basis of down-regulating the expression of p53 in cells, the expression of miR-1469 was up-regulated, and the effect of Forsythin on its apoptosis was detected by Western blot. The results showed that, up-regulating the expression of miR-1469 can reverse the drug inhibition of apoptosis of laryngeal carcinoma cells with low expression of p53. Then, on the basis of down-regulating the expression of p53 in cells, we further down-regulated the expression of Mcl1, and detected the effect of Forsythin on the apoptosis by Western blot. The results showed that, down-regulating the expression of Mcl1 can reverse the inhibitory effect of the drug on the apoptosis of laryngeal carcinoma cells by the low expression of P53. This indicates that Forsythin can up-regulate the expression of miR-1469 through up-regulating the expression of p53. The high expression of miR-1469 can inhibit the expression of its downstream target gene Mcll, and the decrease of Mcll expression promotes the apoptosis of laryngeal carcinoma cells, which clarifies that Forsythin regulates the molecular biological mechanism of miRNA-1469 to promote the apoptosis of laryngeal carcinoma cells.

\section{Conclusion}

Forsythin can promote the apoptosis of laryngeal carcinoma cells by up-regulating the expression of miR-1469 and then down-regulating the expression of Mcl1. The drug can up-regulate the expression of miR-1469 by elevating the expression of P53. miR-1469 can promote the apoptosis of laryngeal carcinoma cells by inhibiting the expression of its downstream target gene Mcl1.

\section{Reference}

Chen, G., Zhou, T., Li, Y., Yu, Z., \& Sun, L. (2017). p53 target miR-29c$3 p$ suppresses colon cancer cell invasion and migration through inhibition of PHLDB2. Biochemical and Biophysical Research Communications, 487(1), 90-95. http://dx.doi.org/10.1016/j. bbrc.2017.04.023. PMid:28392396.

Jaiswal, N., Akhtar, J., Singh, S. P., \& Ahsan, F. (2019). An Overview on Genistein and its Various Formulations. Drug Research (Stuttgart), 5(Dec), 305-313. http://dx.doi.org/10.1055/a-0797-3657. PMid:30517965.
Liu, J., Wang, C., Liu, X., Wang, Y., Liu, H., Ren, G., Zhu, L., Sun, Z., \& Chen, Z. (2017). Low expression of miR-1469 predicts disease progression and unfavorable post-surgical clinical outcomes in patients with esophageal squamous cell cancer. Oncology Letters, 13(6), 4469-4474. http://dx.doi.org/10.3892/ol.2017.5957. PMid:28588716.

Obid, R., Redlich, M., \& Tomeh, C. (2019). The Treatment of Laryngeal Cancer. Oral and Maxillofacial Surgery Clinics of North America, 31(1), 1-11. http://dx.doi.org/10.1016/j.coms.2018.09.001. PMid:30449522.

Sun, S., Wang, X., Xu, X., Di, H., Du, J., Xu, B., Wang, Q., \& Wang, J. (2017a). MiR-433-3p suppresses cell growth and enhances chemosensitivity by targeting CREB in human glioma. Oncotarget, 8(3), 5057-5068. http://dx.doi.org/10.18632/oncotarget.13789. PMid:27926502.

Sun, X., Xu, M., Liu, H., \& Ming, K. (2017b). MicroRNA-219 is downregulated in non-small cell lung cancer and inhibits cell growth and metastasis by targeting HMGA2. Molecular Medicine Reports, 16(3), 3557-3564. http://dx.doi.org/10.3892/mmr.2017.7000. PMid:28714014.

Sun, Y., Wang, F., Wang, L., Jiao, Z., Fang, J., \& Li, J. (2017c). MicroRNA-433 regulates apoptosis by targeting PDCD4 in human osteosarcoma cells. Oncology Letters, 14(2), 2353-2358. http://dx.doi.org/10.3892/ ol.2017.6441. PMid:28781674.

Tron, A. E., Belmonte, M. A., Adam, A., Aquila, B. M., Boise, L. H., Chiarparin, E., Cidado, J., Embrey, K. J., Gangl, E., Gibbons, F. D., Gregory, G. P., Hargreaves, D., Hendricks, J. A., Johannes, J. W., Johnstone, R. W., Kazmirski, S. L., Kettle, J. G., Lamb, M. L., Matulis, S. M., Nooka, A. K., Packer, M. J., Peng, B., Rawlins, P. B., Robbins, D. W., Schuller, A. G., Su, N., Yang, W., Ye, Q., Zheng, X., Secrist, J. P., Clark, E. A., Wilson, D. M., Fawell, S. E., \& Hird, A. W. (2018). Discovery of Mcl-1-specific inhibitor AZD5991 and preclinical activity in multiple myeloma and acute myeloid leukemia. Nature Communications, 9(1), 5341. http://dx.doi.org/10.1038/s41467-01807551-w. PMid:30559424.

Yang, L., Peng, F., Qin, J., Zhou, H., \& Wang, B. (2017a). Downregulation of microRNA-196a inhibits human liver cancer cell proliferation and invasion by targeting FOXO1. Oncology Reports, 38(4), 2148-2154. http://dx.doi.org/10.3892/or.2017.5873. PMid:28791406.

Yang, Y., Zhao, Z., Hou, N., Li, Y., Wang, X., Wu, F., Sun, R., Han, J., Sun, H., Song, T., Huang, C., \& Shao, Y. (2017b). MicroRNA-214 targets Wnt3 to suppress liver cancer cell proliferation. Molecular Medicine Reports, 16(5), 6920-6927. http://dx.doi.org/10.3892/ mmr.2017.7483. PMid:28901526.

Zhang, J., Zhang, L., Zhang, T., Dong, X. M., Zhu, Y., \& Chen, L. H. (2018a). Reduced miR-433 expression is associated with advanced stages and early relapse of colorectal cancer and restored miR-433 expression suppresses the migration, invasion and proliferation of tumor cells in vitro and in nude mice. Oncology Letters, 15(5), 7579-7588. http://dx.doi.org/10.3892/ol.2018.8275. PMid:29740483.

Zhang, T., Jiang, K., Zhu, X., Zhao, G., Wu, H., Deng, G., \& Qiu, C. (2018b). miR-433 inhibits breast cancer cell growth via the MAPK signaling pathway by targeting Rap1a. International Journal of Biological Sciences, 14(6), 622-632. http://dx.doi.org/10.7150/ ijbs.24223. PMid:29904277.

Zhang, Y., Fang, J., \& Zhao, H. (2018c). Downregulation of microRNA-1469 promotes the development of breast cancer via targeting HOXA1 and activating PTEN/PI3K/AKT and $\mathrm{Wnt} / \beta$-catenin pathways. Journal of Cellular Biochemistry, 15, 1-11. http://dx.doi.org/10.1002/ jcb.27786. PMid:30320894.

Zhao, Y., He, J., Li, J., Peng, X., Wang, X., Dong, Z., Zhao, E., Liu, Y., Wu, Z., \& Cui, H. (2017). Demethylzeylasteral inhibits cell proliferation and induces apoptosis through suppressing MCL1 in melanoma cells. Cell Death \& Disease, 8(10), e3133. http://dx.doi.org/10.1038/ cddis.2017.529. PMid:29072681. 